\title{
A Structural Property of Solutions to Path Optimization Problems in Random Access Networks
}

\author{
A. A. Kherani \\ Dept. of Comp. Sc. and Engg. \\ Indian Institute of Technology \\ Delhi, India. \\ Email: alam@cse.iitd.ac.in
}

\author{
D. Kumar, E. Altman \\ INRIA \\ B.P. 93, 2004 Route des Lucioles \\ 06902 Sophia Antipolis, France. \\ Email: \{dkumar,altman\}@sophia.inria.fr
}

\begin{abstract}
The inherent nature of the physical setup and transmission mechanism in wireless ad hoc networks with random channel access, results in correlation between the link metrics of adjacent links, when considering path optimization problems. We identify a special structure inherent to the solution of Dynamic Programming (DP) problem arising in such an optimization over paths. According to this structure, the optimal policy tries to equalize the link metrics of adjacent links in a multi-hop route. We validate this structural property with simulations.
\end{abstract}

\section{INTRODUCTION}

${ }^{1}$ The performance characteristics of either wired or wireless networks are measured and analysed using various kinds of metrics. These metrics usually signify certain performance attributes pertaining to the transmission links in the network. We can therefore think of a link metric that defines the performance measure of transmission links in the network. This link metric could either be some kind of a cost like link delay or it could as well be some kind of a reward utility like link lifetime, link rate, etc. Also see [1], [2] for some examples of such metric. In wire-line networks, a link metric usually depends on certain performance attributes of only one of the end nodes (transmitting node). For example the expected delay over a link depends on the queueing delay of only the transmitting node. Whereas, in random access wireless networks, a link metric may depend on attributes associated with both the end nodes. For example, the link rate and link lifetime in a wireless ad hoc network depends on the power levels or battery energy of both the end nodes. For sure, in both wired and wireless networks, a link metric could as well be some independent entity by itself, e.g., propagation delay and channel gain due to multi-path fading.

Due to dependence on both the end nodes, link metrics of two adjacent links in a multi-hop wireless network may depend on attributes of the common node between them and hence they may be correlated. With another point of view, in wireless networks, attributes of multiple nodes may collectively determine properties of a given link, as opposed to wired networks where properties of a link are determined by attributes of only one terminal node. This fundamental difference between wired and wireless networks requires considering significantly

\footnotetext{
${ }^{1}$ This work was sponsored by "The Indo-French Centre for the Promotion of Advanced Research" under CEFIPRA Project\# 2900-IT-1.
}

different approaches when finding an optimal path between a source node and its destination. It is to be noted that the above discussion is valid only for the choice of those link metric that satisfy the properties discussed in the previous paragraph.

All routing algorithms aim to achieve path optimization, and most of these algorithms use the Dynamic Programming (DP) technique to compute optimal paths. In wired networks, whether the decision is based on a link metric or attributes of nodes, the complexity of finding an optimal path is the same. However, in wireless networks the situation may be very different, especially when the link metric depends on the attributes of both end nodes as discussed before. In wireless networks that have rapidly changing network topologies and require frequent discovery of new routes, getting some insight into the structural properties of the optimal path can help in significantly reducing the time complexity of route rediscovery. This is especially beneficial when nodes are energylimited devices. Our main contributions in this paper are

1) Identify basic difference between path optimization in wired and wireless networks.

2) When the link metric depends on attributes of both the end nodes (i.e., the wireless case), we identify in Section II-B a special structural property that is satisfied by the solution of the DP problem of Section II. This structural property, which says that the optimal policy tends to equalize the link metrics of two successive links, results in significant reduction in the computational complexity of the DP algorithm. In Section III, we validate this structural property by considering a specific example of routing in a vehicular ad hoc network (VANET).

3) Yet another interesting result obtained in this paper is that an optimization problem of a Max-Min form can be transformed to a linear optimization problem (Theorem 2.4).

\section{The BAsic Problem Formulation}

In multi-hop wireless networks, one can divide the problem of finding an optimal path between a source and its destination, into two sub problems:

1) For a given number of intermediate relay nodes, find the best path i.e., the optimal choice of relay nodes that 
optimizes a certain objective function comprising of link metrics, and

2) Having solved the above problem, find the optimal number of relay nodes.

In the present work we are interested in the first optimization problem mentioned above. Since the source and destination nodes are known in advance and thus fixed, we first present the basic problem formulation in a general and natural framework of dynamic programming with fixed initial and terminal states. Let $l_{i}$ denote an attribute associated with node $i$. An example of such an attribute is the (inverse of) average transmit queue occupancy of a node. Let the link metric of a link between nodes $i$ and $j$ be denoted by $H\left(l_{i}, l_{j}\right)$ for some function $H(\cdot, \cdot)$. In the rest of the paper, attributes of a node will also be referred to as node metric. Now, for a given number of intermediate relay nodes $M$, the problem is to find a vector of indices of the relay nodes $\left(i_{1}, \ldots, i_{M}\right)$ that minimizes

$$
\sum_{j=1}^{M+1} H\left(l_{i_{j-1}}, l_{i_{j}}\right)
$$

where the source node has index $i_{0}$ and its destination has index $i_{M+1}$. This kind of an objective function arises for example when we want to minimize the path-sum delay in a route. Before proceeding further, we mention here that the above optimization problem is general enough for also studying the Max-Min form class of problems (this equivalence is shown later), where the objective is to maximize

$$
\min _{j=1, \ldots, M+1} T\left(l_{i_{j-1}}, l_{i_{j}}\right) .
$$

This kind of an objective function may arise, say when we want to maximize (a critical reward) the least of the lifetimes of links constituting a route. Here we use the notation $T(\cdot, \cdot)$ for link metric instead of the one used in Equation 1, for notational convenience. By applying an appropriate transformation to the link metric $H(\cdot, \cdot)$, this will also allow us to solve the problem of Equation 1 in an indirect manner, by using the solution of yet another set of problems that are equivalent to the above Max-Min problem.

In the rest of this section, we first establish the equivalence of the optimization problem of Equation 1 to the Max-Min optimization problem, via yet another set of optimization problems equivalent to the Max-Min problem. Then later in Section II-B we derive a special structural property that is satisfied by the solutions of all these equivalent problems.

\section{A. Equivalence of Equation 1 problem to the Max-Min prob- lem}

The Max-Min optimization problem can be detailed as follows. Let the node metric be allowed to vary over a finite set of values of cardinality $L$. We are given the number of intermediate nodes $M$ in the route path, for a particular choice of a source node and its destination. We want to find the node metrics $l_{i_{j}}, 1 \leq j \leq M$ of the $M$ intermediate relay nodes such that we obtain a maximum value of (a critical reward) the least of the link metrics, i.e., we seek a vector $l_{i_{j}}, 1 \leq j \leq M$ that solves the following optimization problem

$$
\underset{1 \leq l_{i_{j}} \leq L, j=0, \ldots, M}{\operatorname{Maximize}} \underset{j=0, \ldots, M}{\operatorname{Minimum}} T\left(l_{i_{j}}, l_{i_{j+1}}\right) .
$$

This problem can be rewritten as finding a vector $l_{i_{j}}, 1 \leq j \leq$ $M$ that solves the following optimization problem (the only change is that of notation, since here by node $i$ we mean $i^{\text {th }}$ node in the optimal path)

$$
\underset{1 \leq l_{i} \leq L, i=0, \ldots, M}{\operatorname{Maximize}} \underset{i=0, \ldots, M}{\operatorname{Minimum}} T\left(l_{i}, l_{i+1}\right) .
$$

Instead of solving the above problem directly, we further look at a different, parameterized set of objective functions to be optimized. These new objective functions will coincide with the original one of Equation 3 when the parameter takes a special value. Solution to any one of these parameterized form of optimization problems will then finally provide us with the solution to the problem of Equation 1 via the solution to the Max-Min problem of Equation 3. We first report the following simple lemma which we prove here for the sake of completeness.

Lemma 2.1: For any finite dimensional vector $\underline{x}$ with positive elements $x_{i}, 1 \leq i \leq n$, if $\|\underline{x}\|_{\alpha}$ is the $l_{\alpha}$-norm, i.e., $\|\underline{x}\|_{\alpha}=\left[\sum_{1 \leq i \leq n} x_{i}^{\alpha}\right]^{\frac{1}{\alpha}}$, and $\|\underline{x}\|_{\infty}$ denote its $l_{\infty}$-norm, i.e., $\|\underline{x}\|_{\infty}=\max _{1 \leq i \leq n}\left\{x_{i}\right\}$, then

$$
\lim _{\alpha \rightarrow \infty}\|\underline{x}\|_{\alpha}=\|\underline{x}\|_{\infty}
$$

Proof: Let, for the given vector $\underline{x}$,

$$
i^{*} \triangleq \arg \max _{1 \leq i \leq n} x_{i} .
$$

Then, for any $\alpha$,

$$
\|\underline{x}\|_{\infty}=x_{i^{*}} \leq\|\underline{x}\|_{\alpha},
$$

and also,

$$
\|\underline{x}\|_{\alpha} \leq n^{\frac{1}{\alpha}}\|\underline{x}\|_{\infty}
$$

This completes the proof.

Theorem 2.2: The optimization problem of Equation 3 has the same optimizer as that of any of the following optimization problems

$$
\begin{gathered}
\underset{1 \leq l_{i} \leq L, i=1, \ldots, M}{\operatorname{Minimize}}\left[\sum_{j=0}^{M}\left(T\left(l_{j}, l_{j+1}\right)\right)^{-\alpha}\right]^{\frac{1}{\alpha}}, \\
\underset{1 \leq l_{i} \leq L, i=1, \ldots, M}{\text { Maximize }}\left[\sum_{j=0}^{M}\left(T\left(l_{j}, l_{j+1}\right)\right)^{-\alpha}\right]^{\frac{-1}{\alpha}},
\end{gathered}
$$

as $\alpha \rightarrow \infty$.

Proof: The optimization problem of Equation 3 clearly has the same solution as that of the problem

$$
\underset{1 \leq l_{i} \leq L, i=0, \ldots, M}{\operatorname{Minimize}} \underset{i=0, \ldots, M}{\operatorname{Maximum}} \frac{1}{T\left(l_{i}, l_{i+1}\right)} .
$$


Now, for any integer $\alpha>0$, we can compute the $l_{\alpha}$-norm of an $M$-dimensional vector whose $i^{t h}$ element is $1 / T\left(l_{i}, l_{i+1}\right)$. The $l_{\alpha}$-norm of this vector, for any given values of $l_{i}$ 's is

$$
\left[\sum_{i=0}^{M}\left(T\left(l_{i}, l_{i+1}\right)\right)^{-\alpha}\right]^{\frac{1}{\alpha}} .
$$

Since $1 / T\left(l_{i}, l_{i+1}\right)$ 's are strictly positive and bounded quantities, we can invoke Lemma 2.1 to conclude the equivalence of Equations 3 and 4. Then equivalence of Equations 4 and 5 is straightforward.

In fact, we can say something more about the relation between the two optimization problems of Equations 3 and 4. This additional property actually leads us to our main result of this section (Theorem 2.4).

Theorem 2.3: There exists a finite $\alpha^{*}(T)$, such that the maximizer of optimization problem of Equation 3 is same as that of either of Equation 4 or 5, for all values of $\alpha \geq \alpha^{*}(T)$.

Proof: Fix a vector $\underline{x}$ with elements $x_{i}, 1 \leq i \leq n$. Then, from Lemma 2.1 we know that $\lim _{\alpha \rightarrow \infty}\|\underline{x}\|_{\alpha}=$ $\max _{1 \leq i \leq n} x_{i}$. Now, form a vector $y$ whose $i^{t h}$ element $y_{i}$ is the $i^{t h}$ maximum among the elements of $\underline{x}$ (so that $y_{1}=$ $\left.\max _{1 \leq i \leq n} x_{i}=\|\underline{x}\|_{\infty}\right)$. Since the number of elements in $\underline{x}$ is $n$, which is finite, the difference $y_{1}-y_{2}>0$ (assuming that no two elements of $\underline{x}$ are equal; the case where some of the elements of $\underline{x}$ are equal can also be easily considered.). Since $\lim _{\alpha \rightarrow \infty}\|\underline{x}\|_{\alpha}=\lim _{\alpha \rightarrow \infty}\|\underline{y}\|_{\alpha} \rightarrow y_{1}$, for any $\epsilon>0$ there exists a finite $\alpha_{\epsilon}^{*}(\underline{x}, T)$ such that , $y_{1}-\|\underline{x}\|_{\alpha}<\epsilon$ for all $\alpha>\alpha_{\epsilon}^{*}(\underline{x}, T)$.

Now, since the set of possible values of the node metric over which optimization is carried out is finite (of cardinality $L)$, we can define,

$$
\delta \triangleq \min _{l_{i}, l_{j}} \operatorname{POS}\left(\left|T\left(l_{i}, l_{i+1}\right)-T\left(l_{j}, l_{j+1}\right)\right|\right)
$$

where

$$
\operatorname{POS}(|x-y|)= \begin{cases}|x-y| & \text { if }|x-y|>0 \\ |x| & \text { otherwise }\end{cases}
$$

Then, $\alpha^{*}(T) \triangleq \max _{\underline{x}} \alpha_{\delta}^{*}(\underline{x}, T)<\infty$ is the finite quantity that we were seeking.

Theorem 2.3 ensures that there is no discontinuity in the solution to the optimization problem of Equation 4 or 5 as $\alpha \rightarrow$ $\infty$. Working with the objective function of Equation 4 or 5 thus has an advantage that we can work with any finite value of $\alpha$ and, if this solution is independent of $\alpha$, we will have obtained the solution to the optimization problem of Equation 3. Also, the different forms of objective functions in Theorem 2.2 can be used as per convenience depending on whether the link metric is a reward or cost.

Further, the result of Theorem 2.3 can be used to transform the problem of Equation 1 to that of a Max-Min problem:

Theorem 2.4: The minimizer of Equation 1 is same as the optimizer of Equation 3 with $T(\cdot, \cdot)=H(\cdot, \cdot)^{-K}$ for any $K$ satisfying

$$
K \alpha^{*}\left(H^{-K}\right) \leq 1
$$

where $\alpha^{*}(H)$ is the same as in Theorem 2.3.

Proof: We know from Theorem 2.3 that the optimizer of problem

$$
\underset{1 \leq l_{i} \leq L, i=0, \ldots, M}{\operatorname{Maximize}} \underset{i=0, \ldots, M}{\operatorname{Minimum}}\left(H\left(l_{i}, l_{i+1}\right)\right)^{-K}
$$

is the same as the minimizer of Equation 4 (with $\alpha$ replaced by $\beta$ for notational convenience),

$$
\sum_{j=1}^{M+1}\left(H\left(l_{i_{j-1}}, l_{i_{j}}\right)\right)^{K \beta}
$$

for all $\beta \geq \alpha^{*}\left(H^{-K}\right)$. If for some $K$, it turns out that $K \beta=1$ for some $\beta \geq \alpha^{*}\left(H^{-K}\right)$ then the second problem above is that of minimizing Equation 1. A value of $K$ gives rise to existence of such a $\beta$ iff $K \alpha^{*}\left(H^{-K}\right) \leq 1$.

Theorem 2.4 and 2.2 say that for any problem of the kind in Equation 1 we can solve a transformed problem of Equation 3 and vice versa. However, existence of such a value of $K$ is not entirely obvious and needs a proof that follows. We first state the following lemma.

Lemma 2.5: $\alpha^{*}\left(H^{-K}\right) \rightarrow K \rightarrow 01$.

Proof: With $\alpha=1$, as $K \rightarrow 0$, the solution set (the set of optimizers) of Equation 4 converges to that of Equation 3 because the objective function converges to a constant.

Theorem 2.6: There exists a $K$ that satisfies Equation 6.

Proof: The proof follows from Lemma 2.5.

Now, we can use a circular at first sight argument: In order to study the structural property of the minimizer of Equation 1 (with a link metric function $H(\cdot, \cdot)$ ), we solve the equivalent Max-Min problem as in Theorem 2.4 (with a link metric function $\left.H(\cdot, \cdot)^{-K}\right)$. This Max-Min problem is in turn solved using the approach of Theorem 2.2, i.e., by solving problem of Equation 4, again with a link metric function $H(\cdot, \cdot)^{-K}$ and with $\alpha \rightarrow \infty$.

\section{B. Structural property at optimal point}

Consider two nodes, the source node has metric $l_{0}$ and its destination node has metric $l_{M+1}$. Thus, we need to find a set of node metrics $l_{i}, 1 \leq i \leq M$ such that objective function of Equation 4 is maximized. The constraint is that the set of possible values of $l_{i}$ is finite and of cardinality $L$. We see that the objective function for any given value of $\alpha$, for some appropriate function $T(\cdot, \cdot)$, is given by

$$
\left[\sum_{j=0}^{M}\left[\frac{1}{T\left(l_{j}, l_{j+1}\right)}\right]^{\alpha}\right]^{\frac{1}{\alpha}} .
$$

Let $f_{i}(x, y):=\frac{1}{T(x, y)}$ be the link metric of $i^{\text {th }}$ link, if $l_{i}=x$ and $l_{i+1}=y$. Clearly, if one is allowed to choose any value for the intermediate node's attributes (thus not restricting to the finite set of cardinality $L$ ), we would require the metric of node $i$ to be a value $x$ such that

$$
\frac{d}{d x}\left[\left(f_{i-1}\left(l_{i-1}, x\right)\right)^{\alpha}+\left(f_{i}\left(x, l_{i+1}\right)\right)^{\alpha}\right]=0 .
$$


This implies, in particular, that

$$
\frac{f_{i-1}\left(l_{i-1}, x\right)}{f_{i}\left(x, l_{i+1}\right)}=\left[-\frac{d f_{i}\left(x, l_{i+1}\right)}{d f_{i-1}\left(l_{i-1}, x\right)}\right]^{\frac{1}{\alpha-1}} .
$$

Taking $\alpha \rightarrow \infty$, we see that we need

$$
\frac{f_{i-1}\left(l_{i-1}, x\right)}{f_{i}\left(x, l_{i+1}\right)}=1,
$$

implying that the link metrics of successive links should be equalized in order to optimize the objective function of Equation 4. Note that this is only a necessary condition and not a sufficient one, i.e., not all configurations that result in identical link metrics of successive links will be a solution to the optimization problem under consideration. However, any solution to the optimization problem will satisfy the above mentioned property. The above structure also carries over to the case where the allowed values of metrics of the intermediate nodes are restricted to a finite discrete set. However in that case, as it is obvious, exact equalization of the link metric of successive links will not be achieved owing to the lack of choice of metrics for the intermediate nodes. In Section III we consider an example network, where owing to a finite set of possibilities, the optimal policy has to settle down for a convex combination, instead of an exact equalization. It is important to note here that, owing to Theorem 2.4 , the above structural result holds good for both the optimization problems of Equation 1 and Equation 3, as well.

\section{VALIDATION OF EQUALIZATION PROPERTY USING A VANET SIMULATOR}

In order to validate the equalization of link metrics property derived in the previous section, we have developed a vehicular mobile ad hoc network (VANET) simulator. The simulator simulates nodes (vehicles) moving on a highway in their respective lanes and the objective is to find the best path that maximizes the least of the link lifetimes of a route between any given source-destination pair of vehicular nodes.

Each lane is characterized by an associated speed and every node moving in a given lane travels with this same speed that is associated with its lane. Thus all vehicles moving in the same lane have identical speeds. The vehicles can change lanes and hence speeds, randomly over time. Each node is assumed to have a motion that is independent of the other nodes. To be more precise, we assume that the stochastic process corresponding to the changing speed of a node, is a Markov chain over the set of different speeds associated with different lanes on the highway. The time is slotted and at the end of each time slot each node makes a decision on whether to change its lane or not. This decision is made independently of any past decisions. If a node decides to change its lane, it moves to either of its adjacent lanes according to some probability distribution. Thus a node continues to move in a particular lane for a random amount of time that is geometrically distributed. We assume that a node in lane $i, 1 \leq i \leq L$, transits to any of the adjacent lanes at the beginning of a time slot with probability $1-p_{i}$.
We assume that such a mobile ad hoc network formed by vehicles moving on a highway consists of a dense layout of nodes, so that there is at least one node inside any sufficiently small area on any given lane. The problem that we address is to find the best path that maximizes the lifetime of a route between any given source-destination pair of nodes that can not communicate directly, thus requiring relaying by intermediate nodes. We assume that the speed (or, the lane) of the source and its destination and the initial distance $D$ between them (at the time of route setup) are given. Also given is $M$, the number of intermediate relay nodes and the hop distances $d_{i}, 1 \leq i \leq M$ between these relay nodes at the time of route setup; thus the initial distance $\left(d_{M+1}\right)$ between the last relay node and the destination node is fixed since $D$ is given. The distances between these $M$ relay nodes can change with time if they are moving on lanes with different speeds. Hence the problem is to find the speeds of the intermediate nodes so as to maximize the time until when any one of the links constituting the whole route, first breaks down, i.e., maximize the route lifetime or in other words maximize the minimum of expected link lifetimes which is the link metric here. Therefore, if $v_{i}, 0 \leq i \leq M+1$ is the initial speed of $i^{\text {th }}$ node in the path $(0$ and $M+1$ indices are for source and destination nodes respectively), and if $T\left(d_{i}, v_{i-1}, v_{i}\right)$ denotes the expected time until when the nodes $i-1$ and $i$ can communicate, then the problem becomes

$$
\operatorname{Maximize}_{v_{1}, \ldots, v_{M}} \operatorname{Minimum}_{1 \leq i \leq M+1} T\left(d_{i}, v_{i-1}, v_{i}\right) .
$$

With respect to our basic problem of Section II, the link metric here is $T(\cdot, \cdot, \cdot)$ and the node attribute or node metric here is the speed $v_{i}$ of a node. Thus, for a given value of the hop distances, the above problem is identical to that of Equation 3 in Section II.

In the simulation results presented here, the time slot length has been taken as 0.1 seconds. For our simulations we consider that the probabilities $p_{1}=\cdots=p_{L}=p$ are identical for all lanes. The set of possible lane speeds is $s_{i}, 1 \leq i \leq L$ and hence $v_{j} \in\left\{s_{i}, 1 \leq i \leq L\right\}$. We assume that $s_{1}<$ $s_{2}<\cdots<s_{L}$. The simulator computes the expected link lifetimes of all possible links by exhaustively simulating over all possible speed assignments $\underline{v}=\left\{v_{1}, \cdots, v_{M}\right\}$ to the relay nodes for a given scenario of $M$ relay nodes, $L$ lanes, the hop distance vector $\underline{d}=\left\{d_{1}, \cdots, d_{M}\right\}$, speeds of source and destination $v_{0}$ and $v_{M+1}$, wireless transmission range $R$, source and destination separation $D$ and the probability $p$. Once an exhaustive set of link lifetimes for all possible values of $\underline{v}$ is obtained by employing this brut force method, either of the objective functions of Equation 3 or 4 is applied over this set to obtain an optimal speed assignment policy resulting in the choice of a best or optimal path.

\section{A. Simulation scenarios}

A car battery operated mobile device has a typical transmission range of around 200 meters. We therefore consider hop distances in a VANET to vary from 140 to 200 meters and a wireless transmission range of 200 meters is considered for 


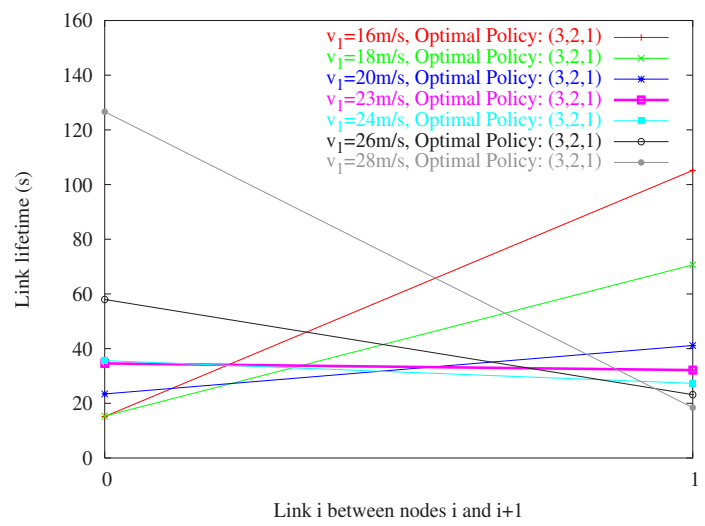

Fig. 1. Link lifetime equalization over continuum of speeds

all the simulation scenarios. It has been shown in a previous work [3] that large number of hops in an ad hoc network can significantly degrade the TCP throughput performance. Based on this result, we consider the number of hops $(M+1)$ to vary from 2 to 7 only and the distance between the source and destination nodes is varied from 800 to 1200 meters. We perform simulations for the number of lanes $L$ varying from 2 to 6 and unless explicitly stated in the discussion on the simulation results, the associated speeds are chosen as shown in the table that follows,

\begin{tabular}{|c|c|c|c|c|c|c|}
\hline$l$ & 1 & 2 & 3 & 4 & 5 & 6 \\
\hline$s_{l}(\mathrm{~m} / \mathrm{s})$ & 14 & 17 & 22 & 30 & 42 & 55 \\
\hline$\approx s_{l}(\mathrm{~km} / \mathrm{hr})$ & 50 & 60 & 80 & 110 & 150 & 200 \\
\hline
\end{tabular}

Simulations were carried out for a large set of real life scenarios. In the following part of this section we discuss the various scenarios that were simulated and compare their results with the link metric equalization property that we obtained in this paper earlier.

1) Lifetime Equalization over continuum set of speeds for small transition probabilities: In Figure 1 we plot the results of the scenario $L=3, M=1, v_{0}=s_{3}=22 \mathrm{~m} / \mathrm{s}, v_{2}=s_{1}=$ $14 \mathrm{~m} / \mathrm{s}, p=0.99999, D=300 \mathrm{~m}$ and $\underline{d}=(143,157)$. In order to be able to validate the equalizing structure obtained earlier over a continuum set of intermediate node speeds, we vary the speed associated with lane 2 from $14 \mathrm{~m} / \mathrm{s}$ to $30 \mathrm{~m} / \mathrm{s}$ in small steps of $2 \mathrm{~m} / \mathrm{s}$ and plot the link lifetimes for each such speed of lane 2 separately. This allows the only intermediate node 1 to be assigned with one of the quasi-continuum set of speeds for the optimization problem of Equation 3 or 4 equivalently. It can be seen in the figure that under optimality, for varying values of $v_{1}$, the optimal lifetimes of the links between node 0 and 1 and node 1 and 2 are different. However for $v_{1}=23 \mathrm{~m} / \mathrm{s}$ the optimal lifetimes of the two adjacent links are almost equal (the line joining them is almost flat) thus confirming our result obtained in Section II-B that the lifetimes of adjacent links should be equalized in order to optimize the objective function of Equation 4. In fact, it can be observed that we obtain the maximum of the least of the

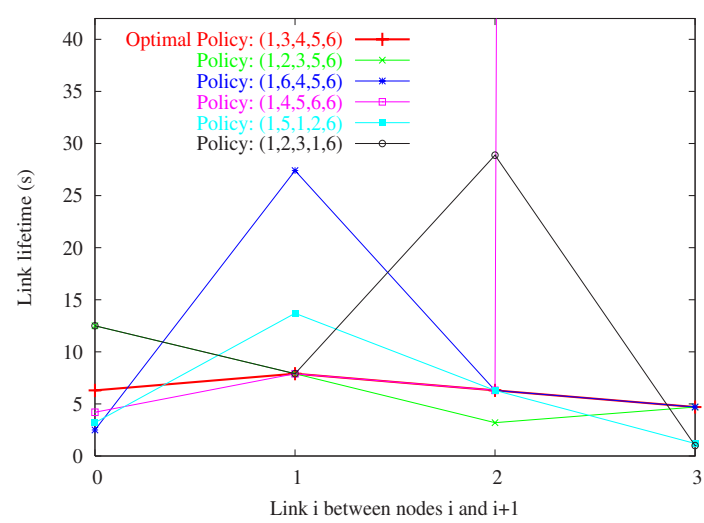

Fig. 2. Link lifetime equalization under optimal policy

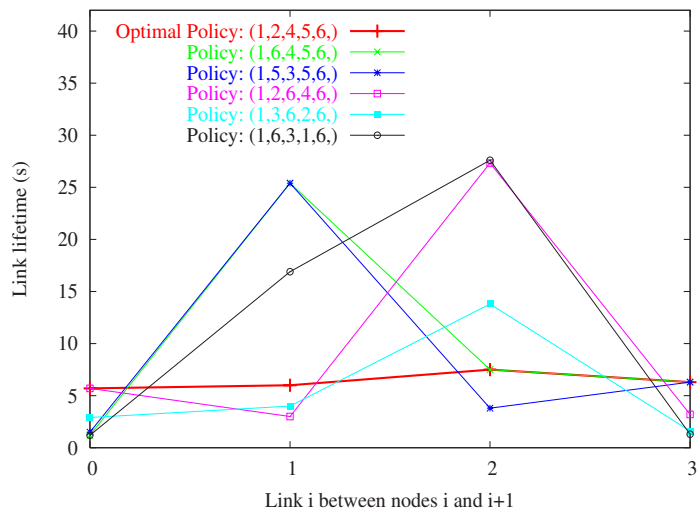

Fig. 3. Link lifetime equalization under optimal policy

two link lifetimes for speed $v_{1}=23 \mathrm{~m} / \mathrm{s}$ and the optimal lifetimes obtained for other values of $v_{1}$ are not truly optimal because of the unavailability of the choice of speed $23 \mathrm{~m} / \mathrm{s}$ in those cases.

2) Lifetime Equalization under the optimal policy for small transition probabilities: To strengthen our validation of the link metric equalization structure discussed in the previous note, in Figure 2, we plot the link lifetimes for a particular subset of the all possible speed combinations (including the optimal combination) for a given scenario. Figures 3 and 4 illustrate the same result for two more set of given scenarios. It is clearly seen in these figures that at the optimal policy the link lifetimes are equalized and the maximum of the least link lifetimes is obtained at the optimal policy, by definition. Figure 2 uses the scenario $L=6, M=3$, $v_{0}=14 \mathrm{~m} / \mathrm{s}, v_{4}=30 \mathrm{~m} / \mathrm{s}, p=0.99999, D=700 \mathrm{~m}$ and $\underline{d}=(160,175,180,185)$. Figure 3 uses the same scenario as in Figure 2 except for $\underline{d}=(182,162,176,180)$. Figure 4 uses the scenario $L=55, M=3, v_{0}=14 \mathrm{~m} / \mathrm{s}, v_{4}=30 \mathrm{~m} / \mathrm{s}$, $p=0.99999, D=700 m$ and $\underline{d}=(169,166,167,198)$.

\section{CONCLUSION}

We have considered path optimization problems in wireless networks where a link metric depends on attributes associated with the end nodes. We relate these path optimization problems 


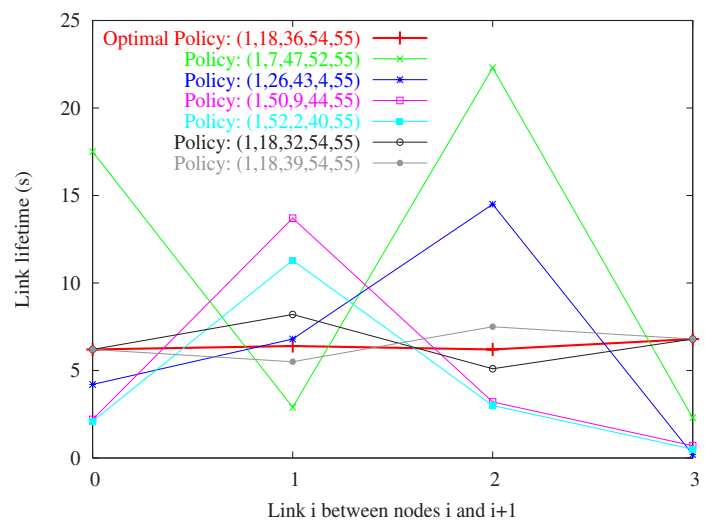

Fig. 4. Link lifetime equalization under optimal policy

to a Max-Min form class of problems and show that the optimal or best path satisfies the property that the link metrics of consecutive links in a multi-hop route are equalized. This property has been validated with a vehicular mobile ad hoc network (VANET) simulator for the route lifetime maximization problem.

\section{REFERENCES}

[1] R. Draves, J. Padhye and B. Zill. "Routing in Multi-radio, Multi-hop Wireless Mesh Network", MOBICOM 2004.

[2] R. Draves, J. Padhye and B. Zill. "Comparison of Routing Metrics for Multi-Hop Wireless Networks", SIGCOMM 2004.

[3] M. Gerla, K. Tang and R. Bagrodia. "TCP Performance in Wireless Multihop networks", IEEE WMCSA, Feb, 1999.

[4] D. Johnson, D. Maltz and J. Broch, "DSR: The Dynamic Source Routing Protocol for Multi Hop Wireless Ad Hoc Networks", In Ad Hoc Networking, Edited by Charles E. Perkins, Addision-Wesley, 2001. 